\title{
Improvement of Interior Ballistic Performance Utilizing Particle Swarm Optimization
}

\author{
Hazem El Sadek, Xiaobing Zhang, Mahmoud Rashad, and Cheng Cheng \\ School of Energy and Power Engineering, Nanjing University of Science and Technology, Nanjing 210094, China \\ Correspondence should be addressed to Hazem El Sadek; zozaka2002@yahoo.com
}

Received 22 January 2014; Revised 23 February 2014; Accepted 23 February 2014; Published 6 April 2014

Academic Editor: Robertt A. Fontes Valente

Copyright (C) 2014 Hazem El Sadek et al. This is an open access article distributed under the Creative Commons Attribution License, which permits unrestricted use, distribution, and reproduction in any medium, provided the original work is properly cited.

\begin{abstract}
This paper investigates the interior ballistic propelling charge design using the optimization methods to select the optimum charge design and to improve the interior ballistic performance. The propelling charge consists of a mixture propellant of seven-perforated granular propellant and one-hole tubular propellant. The genetic algorithms and some other evolutionary algorithms have complex evolution operators such as crossover, mutation, encoding, and decoding. These evolution operators have a bad performance represented in convergence speed and accuracy of the solution. Hence, the particle swarm optimization technique is developed. It is carried out in conjunction with interior ballistic lumped-parameter model with the mixture propellant. This technique is applied to both single-objective and multiobjective problems. In the single-objective problem, the optimization results are compared with genetic algorithm and the experimental results. The particle swarm optimization introduces a better performance of solution quality and convergence speed. In the multiobjective problem, the feasible region provides a set of available choices to the charge's designer. Hence, a linear analysis method is adopted to give an appropriate set of the weight coefficients for the objective functions. The results of particle swarm optimization improved the interior ballistic performance and provided a modern direction for interior ballistic propelling charge design of guided projectile.
\end{abstract}

\section{Introduction}

Recently, study of the propelling charge design becomes very essential to achieve the interior ballistic performance and assure the safety firing. This study is known as design of the interior ballistic which is considered as a crucial branch of gun system design. There are a number of computer-based interior ballistic models with different capabilities. These models allow the researchers of interior ballistic to predict the interior ballistic performance of a particular gun, charge, and projectile combination. The classic interior ballistics models including the characteristics of the gun, charge, and projectile are utilized to predict the muzzle velocity and the peak pressure. But these models cannot provide the best design of the propelling charge that gives the optimum solution of the interior ballistic performance.

Optimization techniques allow the designers to evaluate a large number of design alternatives in a systematic and efficient manner to find the best design. The solution of an interior ballistic model is considered improved if there is a net increase in muzzle velocity without violating gun constraints. The optimization techniques are used to improve the gun performance and/or decrease the design cost while meeting the constraints appropriate to the problem. Therefore, some researchers tried to apply the optimization techniques with the interior ballistic models. A numerical optimization method called augmented Lagrange multiplier was carried out and coupled to a classic interior ballistic model. This method was used to design the parameters of seven-perforation propellant and to obtain the best muzzle velocity for the projectile fired from $120 \mathrm{~mm}$ tank cannons [1]. Another optimization technique was carried out to optimize the ignition system and propelling charge structure in a high muzzle velocity gun [2]. Some researchers of our group tried to use the genetic algorithm (GA) [3]. This algorithm is considered as one of the most currently popular intelligent optimizations to optimize the interior ballistic problem. GA was introduced as a computational analogy of adaptive systems. It is modeled loosely on the principles of the evolution via natural selection, employing a population 
of individuals that undergo selection in the presence of variation-inducing operators such as mutation and recombination (crossover). A fitness function is used to evaluate individuals, and reproductive success varies with fitness. In GA, each genome (chromosome) contains information of the optimization problem by encoding and then should decode the genome information to the optimization problem after finding the optimum solution. According to these above trials of optimization techniques, it is found that they have some limitations in the accuracy of the solution and speed of the convergence. Moreover, the interior ballistic problem cannot be adjusted in a good manner and sometimes the optimum solution cannot be reached.

Particle swarm optimization (PSO) was firstly developed by Kennedy and Eberhart [4]. It was considered as an expansion of an animal social behavior simulation system that incorporated concepts such as nearest-neighbor velocity matching and acceleration by distance. The PSO technique becomes very popular due to its ease of implementation and the quick convergence to the optimum solution [5, 6]. Similarly to evolutionary algorithms, PSO exploits a population (a swarm) of potential solutions (particles) which are modified stochastically at each iteration of the algorithm. However, the manipulation of swarm differs significantly from that of evolutionary algorithms, promoting a cooperative rather than a competitive model. More specifically, the other evolutionary algorithms use explicit mutation and selection operators, while PSO uses an adaptable velocity vector for each particle in order to modify the population and favor the best performing individuals. The velocity vector is used to update the position of the particles at each iteration. The particles are moving towards promising regions of the search space by exploiting information springing from their own experience during the search, as well as the experience of other particles. For this purpose, a separate memory is used where each particle stores the best position it has ever visited in the search space.

In this work, PSO technique is coupled with the interior ballistic model to optimize the propelling charge design. The utilized charge is a mixture charge which consists of two different propellants, granular seven-perforated propellant and tubular propellant. PSO technique is applied to the classic interior ballistic model for $76 \mathrm{~mm}$ naval gun with guided projectile to improve the interior ballistic performance. Two types of objective functions are used with PSO techniques, single-objective function and multiobjective function. Through this work, it is found that the optimization results improved the interior ballistic performance and firing safety with a high quality and quick convergence of the optimum solution.

\section{Problem Formulation}

The optimization of the charge design in interior ballistic process is considered as nonlinear optimization, multiconstrained problem. It consists of multiobjective function and multiple design variables. The design variables have different effects on the performance of interior ballistic. In the interior ballistic process coupled with the optimization technique, there are restrictions between the objective functions. For example, the designer of the interior ballistic intends to obtain the maximum muzzle velocity as a main objective. Maximum muzzle velocity requires a high muzzle pressure, while one of the objective functions is to minimize the muzzle pressure. Hence, the restriction is clear between the muzzle velocity objective function and muzzle pressure objective function. The objective functions should be weighted according to the advantages and disadvantages. The general optimization problem for the classical interior ballistic process can be described as follows:

$$
\begin{array}{cl}
\text { Minimize } & f(x)=\left(f_{1}(x), f_{2}(x), \ldots, f_{k}(x)\right) \\
\text { subject to } & g_{i}(x) \leq 0 \quad \forall i=1,2, \ldots, m \\
& h_{j}(x)=0 \quad \forall j=1,2, \ldots, p \\
& x_{l}^{L} \leq x_{l} \leq x_{l}^{U} \quad \forall l=1,2, \ldots, q,
\end{array}
$$

where $x=\left(x_{1}, x_{2}, \ldots, x_{n}\right)^{T}$ is the decision vector (design variables), $f(x)$ is the multiobjective vector, $f_{k}(x)$ is the $k$ th objective function, $g_{i}(x)$ and $h_{i}(x)$ are inequality and equality constraints, respectively, and $x_{l}^{L}$ and $x_{l}^{U}$ are the lower and upper bound of the design variables, respectively.

The relation between the optimization technique and the interior ballistic model is the objective functions that are calculated from the interior ballistic model and then optimized by the optimization model.

There exist some different tools to simulate the interior ballistic process such as lumped-parameter model and twophase flow model. In this work, lumped-parameter model is utilized to simulate the interior ballistic process and calculate the objective values that will be optimized by using the PSO technique. The lumped-parameter model can be written as follows [7]:

$$
\begin{aligned}
\psi_{i} & =\chi_{i} Z_{i}\left(1+\lambda_{i} Z_{i}+\mu_{i} Z_{i}^{2}\right), \\
\frac{d Z_{i}}{d t} & =\frac{u_{1 i}}{e_{1 i}} p^{n_{i}} \quad i=1,2, \ldots, n, \\
\frac{d v}{d t} & =\frac{S \cdot p}{\varphi \cdot m}, \\
\frac{d l}{d t} & =v \\
S \cdot p\left(l_{\psi}+l\right) & =\sum_{i=1}^{n} f_{i} \omega_{i} \psi_{i}-\frac{k-1}{2} \varphi m v^{2},
\end{aligned}
$$

where $\psi_{i}$ is the relative burnt percentage of the $i$ th propellant, $Z_{i}$ is the relative burnt thickness of the $i$ th propellant, $\chi_{i}, \lambda_{i}$, $\mu_{i}$ are characteristic parameters of the $i$ th propellant, $u_{1 i}$ is the burning rate coefficient of the $i$ th propellant, $e_{1 i}$ is the half web thickness of the $i$ th propellant, $p$ is the pressure in the chamber, $n_{i}$ is the burning rate pressure index of the $i$ th propellant, $\varphi$ is the coefficient accounting for the secondary energy losses, $m$ is the projectile mass, $v$ is the projectile velocity, $S$ is the barrel cross-section area, $l$ is the tube length, 
$l_{\psi}$ is the ratio of chamber free volume to the bore area, $f$ is the impetus force of the $i$ th propellant, $\omega_{i}$ is mass of the $i$ th propellant, and $k$ is propellant specific heat ratio.

According to the form shape function of the sevenperforated propellant, $\psi_{i}$ should be changed at the start point of slivers separation. The form shape function can be described in (3) as follows:

$$
\begin{gathered}
\psi_{i}= \begin{cases}\chi_{i} Z_{i}\left(1+\lambda_{i} Z_{i}+\mu_{i} Z_{i}^{2}\right) & 0 \leq Z_{i}<1 \\
\chi_{s i} Z_{i}\left(1+\lambda_{s i} Z_{i}\right) & 1 \leq Z_{i}<Z_{k i} \\
1 & Z \geq Z_{k i}\end{cases} \\
Z_{k i}=\frac{e_{1 i}+\rho_{i}}{e_{1 i}}
\end{gathered}
$$

\section{Formulation of Particle Swarm Optimization Algorithm}

In the literature there exist numerous methods of optimization that study and analyze the optimization problems under different conditions. PSO is considered as one of the best computationally efficient optimization techniques. It converges to the optimal solution in many problems where most analytical methods fail to converge. PSO has some advantages over other similar optimization techniques, namely, the following [8].

(1) PSO has few and simple parameters. Hence, it is easy to implement.

(2) It has a more effective memory capability than the GA as every particle remembers its own previous best value as well as the neighborhood's best value.

(3) PSO is more efficient in maintaining the diversity of the swarm, since all the particles use the information related to the most successful particle in order to improve themselves, whereas, in GA, the worse solutions are discarded and only the good ones are saved; therefore, in GA the populations evolve around a subset of the best individuals.

The procedure of PSO algorithm can be described as follows.

(1) Initialize the swarm by assigning a random position in the problem hyperspace to each particle (the swarm composed of population of random solutions called particles).

(2) Evaluate the fitness function for each particle (fitness function is the objective function obtained from the interior ballistic simulation).

(3) For each individual particle, particle's fitness value is compared with its $p_{\text {best }}$ (personal best position). If the current value is better than the $p_{\text {best }}$ value, then set this value as the $p_{\text {best }}$ and the current particle's position, $x_{i}$, as $p_{i}$.

(4) Identify the particle that has the best fitness value in the swarm. The value of its fitness function is identified as $g_{\text {best }}$ (global best position) and its position as $p_{g l}$.
(5) Update the velocities and positions of all the particles.

(6) Repeat steps 2-5 until the condition of stopping is met (e.g., maximum number of iterations or a sufficiently good fitness value)

The updating rules of velocity and position can be described as follows:

$$
\begin{aligned}
v_{i, j}(t)= & \omega v_{i, j}(t-1)+c_{1} \operatorname{rand}_{1}\left(p_{i}-x_{i, j}(t-1)\right) \\
& +c_{2} \operatorname{rand}_{2}\left(p_{g l}-x_{i, j}(t-1)\right), \\
x_{i, j}(t)= & x_{i, j}(t-1)+v_{i, j}(t),
\end{aligned}
$$

where $v_{i, j}$ is the velocity of particle $i$ of the $j$ th dimension, $x_{i, j}$ is the position of particle $i$ of the $j$ th dimension, $t$ indicates the iteration number, $i=1,2, \ldots, N, j=1,2, \ldots, M, N$ is the size of swarm, $M$ is the dimension of the search space, $c_{1}$ and $c_{2}$ are the acceleration coefficients (generally $c_{1}+c_{2} \leq 4$ ), $\operatorname{rand}_{1}$ and rand $_{2}$ are random numbers $\in[0,1]$, and $\omega$ is inertia weight which is used as a parameter to control the exploration and exploitation in the search space.

\section{Application of PSO Algorithm to Interior Ballistic Model}

PSO technique is coupled with the lumped-parameter model to improve the interior ballistic performance via optimizing the propelling charge design. This model is applied to $76 \mathrm{~mm}$ naval gun with guided projectile utilizing mixed propellant. Two different approaches will be investigated through this present work:

(1) single-objective function PSO method,

(2) multiobjective function PSO method.

4.1. Single-Objective PSO Method. Firstly, the single-objective PSO method is carried out in order to predict the optimum charge design and to improve the performance of interior ballistic process. The single-objective optimization problem contains objective function, design variables, and constraints. Through this problem the muzzle velocity is considered as the objective function.

4.1.1. Objective Function. The muzzle velocity is considered as the most important parameter in the interior ballistic process. Hence, the only objective function in this method is maximizing the muzzle velocity.

4.1.2. Design Variables. Selection of the design variables is considered very crucial to obtain the optimum solution. Half web thickness and loading density of the propellant are considered the main characteristic parameters of the propellant [3]. In this work, two types of propellants are used. Hence, the design variables are considered as $x=$ $\left[e_{1}, \Delta_{1}, e_{2}, \Delta_{2}\right]$, where $e_{1}$ and $\Delta_{1}$ are half web thickness and loading density of the granular propellant and $e_{2}$ and $\Delta_{2}$ are half web thickness and loading density of the tubular propellant. 
TABLE 1: Nonoptimized parameters.

\begin{tabular}{lccccc}
\hline Parameter & Value & Unit & Parameter & Value & Unit \\
\hline Gun caliber & 0.076 & $\mathrm{~m}$ & Chamber volume & 0.00354 & $\mathrm{~m}^{3}$ \\
Tube length & 4.045 & $\mathrm{~m}$ & Impetus force & 980000 & $\mathrm{~J} / \mathrm{Kg}$ \\
Chamber length & 0.38 & $\mathrm{~m}$ & Ignition temp. & 615 & $\mathrm{~K}$ \\
Projectile mass & 5.9 & $\mathrm{Kg}$ & Co-volume & 0.001 & $\mathrm{~m}^{3} / \mathrm{Kg}$ \\
\hline
\end{tabular}

4.1.3. Constraints. The penalty method is utilized to treat the constraints of the optimization problem. The original constrained problem is replaced by a sequence of unconstrained problems $[9,10]$. The unconstrained problem consists of the objective function and the penalty terms according to each constraint. Penalty terms penalize the objective function when its corresponding constraint is violated.

The selection of the constraints limits is considered according to the launch safety, the technical requirements, the experimental work for charge design, and the data of the typical gun.

The details of the constraints can be described as follows.

(1) Projectile Muzzle Velocity. The minimum value of muzzle velocity $V_{\min }$ is determined according to the sufficient performance required to satisfy the tactical requirements. The muzzle velocity $V_{m}$ should be higher than or at least equal to the $V_{\min }$ :

$$
V_{m} \geq V_{\min }
$$

(2) Maximum Chamber Pressure. According to the launch safety requirements, the strength of the gun tube has a limit. The peak pressure must be lower than the maximum pressure value $P_{m}$; this value is considered a design constant for each gun caliber. Hence, the peak pressure $P_{\max }$ should be lower than this value:

$$
P_{\max } \leq P_{m}
$$

(3) Charge Loading Density. The chamber volume is a fixed value. Hence the propelling charge density has a limit. Consider

$$
0<\Delta<\Delta_{j},
$$

where $\Delta_{j}$ is considered the maximum charge density limit calculated according to the fixed chamber volume.

The loading density limits depend on the launch safety and the required interior ballistic performance. The limits of this constraint can be considered as follows:

$$
0.4 \leq \Delta \leq 0.8 \mathrm{~kg} \cdot \mathrm{dm}^{-3} \text {. }
$$

(4) Relative Charge Burnout Point. Relative burnout point $\eta_{k}$ is considered as an indicator that explains whether the propellant is completely burnt or not. $\eta_{k}$ can be calculated using the following equation:

$$
\eta_{k}=\frac{l_{k}}{l}
$$

where $l$ is the bore length and $l_{k}$ is the projectile travel inside the bore associated with the complete burning of the propellant.

Low relative burnout point means that the propellant was burnt out early while the projectile was still inside the bore. Hence, some of the kinetic energy of the projectile will be lost to overcome the engraving force. Large relative burnout point means that the projectile exited from the muzzle and the propellant was not completely burnt. Hence, the remaining amount of the propellant is considered as a waste propellant. Due to charge design experience, the limits of this constraint can be considered as follows:

$$
0.5 \leq \eta_{k} \leq 0.8
$$

(5) Energy Efficiency of the Charge. Energy efficiency of the charge $\gamma_{g}$ indicates whether the charge is fully utilized. It explains the ratio between the energy delivered to the projectile and the energy of the charge burning. Low value of $\gamma_{g}$ means small kinetic energy delivered to the projectile. $\gamma_{g}$ is calculated using the following equation:

$$
\gamma_{g}=\frac{(1 / 2) m v_{m}^{2}}{f \omega /(k-1)}
$$

The limits of $\gamma_{g}$ can be considered as follows:

$$
0.16 \leq \gamma_{g} \leq 0.6
$$

(6) Half Web Thickness. Values of the half web thicknesses $e_{1}$ and $e_{2}$ of the propellant depend on the gun caliber. The limits of $e_{1}$ and $e_{2}$ should be selected carefully according to the charge manufacturing, launch safety, and technical requirements. These limits can be considered as follows:

$$
\begin{aligned}
& 0.25 \leq e_{1} \leq 0.9 \mathrm{~mm}, \\
& 0.25 \leq e_{2} \leq 0.9 \mathrm{~mm} .
\end{aligned}
$$

The nonoptimized parameters of the interior ballistic process for the $76 \mathrm{~mm}$ gun are tabulated in Table 1. 


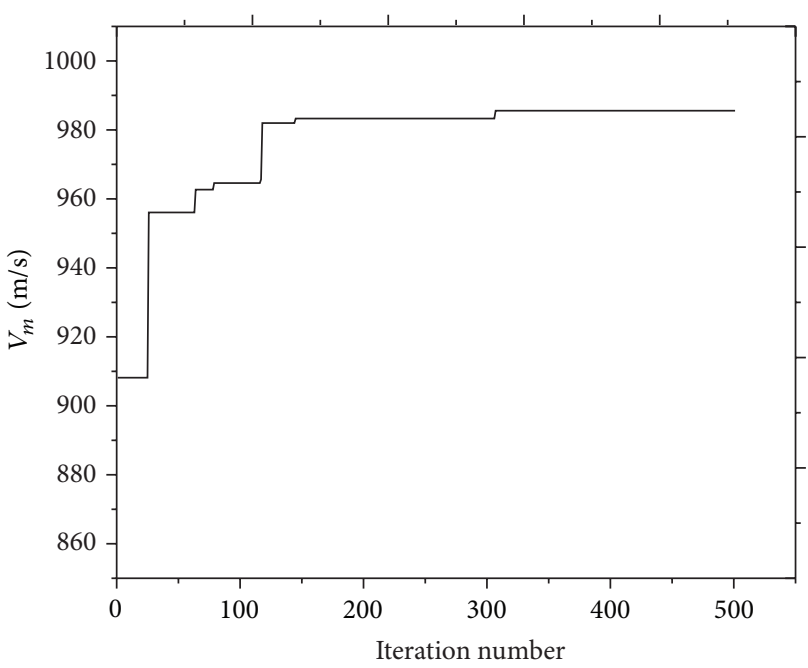

(a) GA

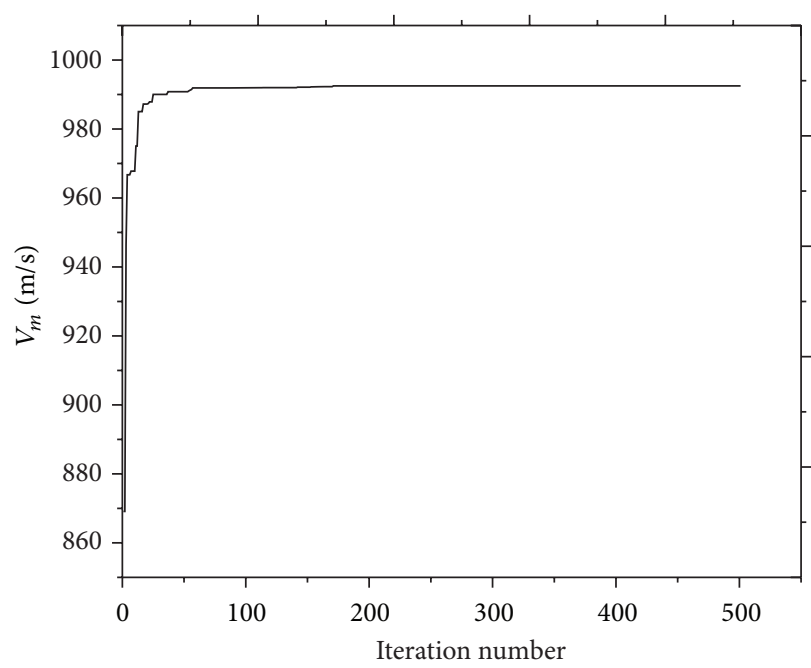

(b) PSO

FIGURE 1: Convergence graph of different algorithms for single-objective problem.

TABLE 2: Parameters of GA.

\begin{tabular}{lccc}
\hline Parameters & Value & Parameters & Value \\
\hline Population size & 40 & Number of constrains & 6 \\
Number of generations & 500 & Probability of crossover & 0.8 \\
Number of objectives & 1 & Probability of mutation & 0.2 \\
\hline
\end{tabular}

TABLE 3: Parameters of PSO.

\begin{tabular}{lccc}
\hline Parameters & Value & Parameters & Value \\
\hline Population size & 40 & Number of constrains & 6 \\
Number of generations & 500 & First acceleration coefficient $c_{1}$ & 1.8 \\
Number of objectives & 1 & Second acceleration coefficient $c_{2}$ & 1.8 \\
\hline
\end{tabular}

In this section, the general optimization model can be written in (1) with the design variables $x=\left[e_{1}, \Delta_{1}, e_{2}, \Delta_{2}\right]$ :

$$
\begin{array}{ll}
\min f(x) & =-V_{m} \\
\text { subject to } & V_{m} \geq 800 \mathrm{~ms}^{-1} \\
& P_{\max } \leq 350 \mathrm{MPa} \\
& 0.4 \leq \Delta \leq 0.8 \mathrm{Kg} \cdot \mathrm{dm}^{-3} \\
& 0.5 \leq \eta_{k} \leq 0.8 \\
& 0.16 \leq \gamma_{g} \leq 0.6 \\
& 0.25 \leq e_{1} \leq 0.9 \mathrm{~mm} \\
& 0.25 \leq e_{2} \leq 0.9 \mathrm{~mm} .
\end{array}
$$

In the single-objective problem, two different optimization techniques, GA and PSO, are carried out in conjunction with the lumped-parameter model. The values of the main parameters utilized in GA and PSO algorithm are tabulated in Tables 2 and 3, respectively.
The optimization results for GA and PSO algorithms compared with the experimental data are tabulated in Table 4. The convergence of the two different algorithms for the objective function is illustrated in Figure 1.

From the optimization results illustrated in Table 4 and Figure 1, it is clear that PSO technique is the winner. PSO provides a better performance of interior ballistic propelling charge design. The better performance is represented in the speed of convergence (PSO converges after 170 iterations, while GA converges after 307 iterations) and the solution quality $\left(V_{m}=992.52 \mathrm{~m} / \mathrm{s}\right.$ in PSO, while $V_{m}=985.8 \mathrm{~m} / \mathrm{s}$ in GA).

Figure 2 represents the convergence of different parameters of the interior ballistic obtained by using PSO technique. These parameters include the design variables and some interior ballistic performance indexes.

4.2. Multiobjective PSO Method. In the actual design process, the designers have different interests in the objective function. Hence, the multiobjective model should be considered 


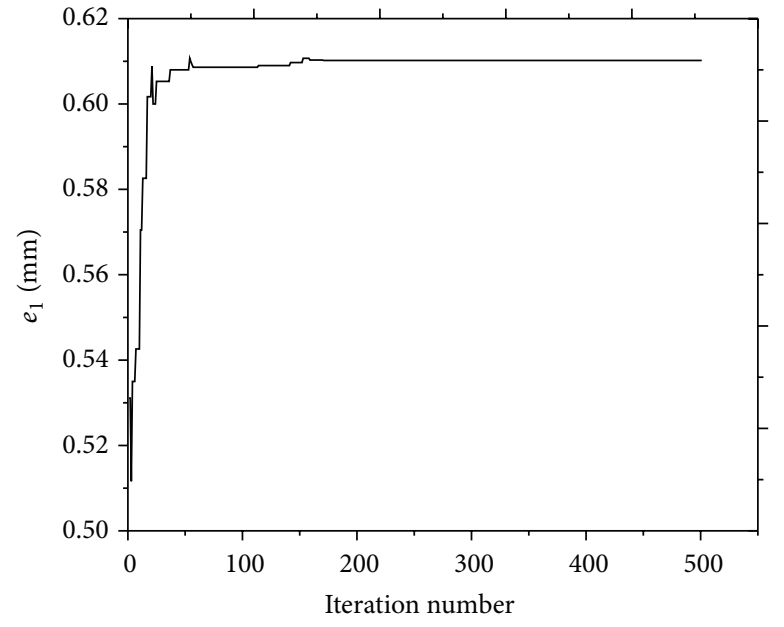

(a) $e_{1}(\mathrm{~mm})$

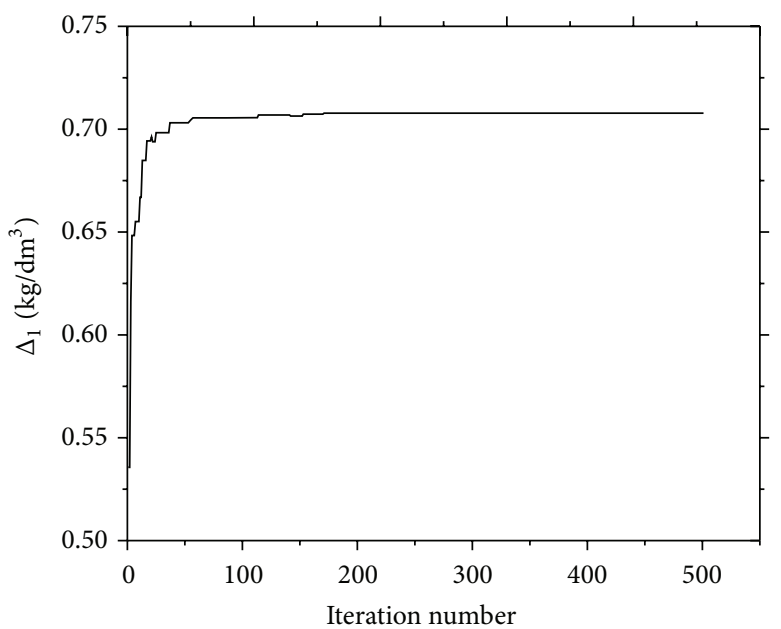

(c) $\Delta_{1}\left(\mathrm{~kg} / \mathrm{dm}^{3}\right)$

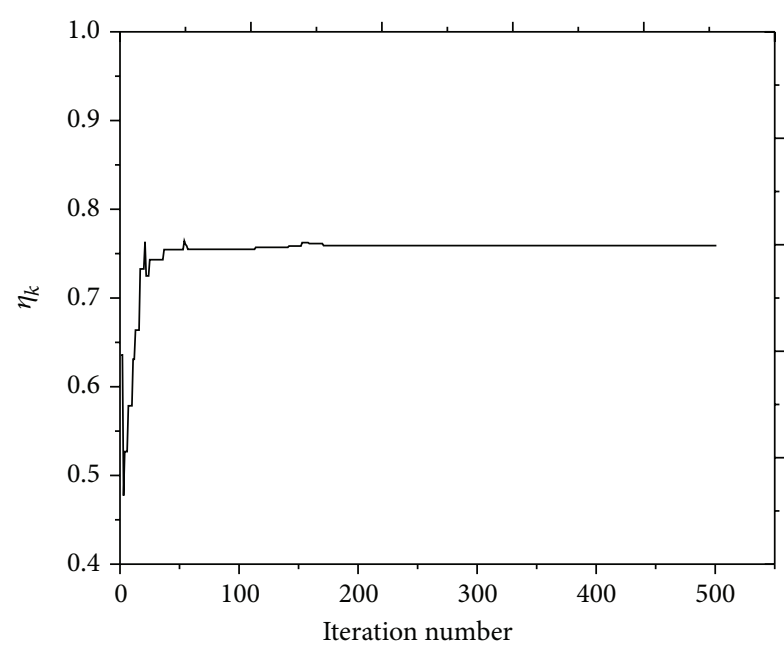

(e) $\eta_{k}$

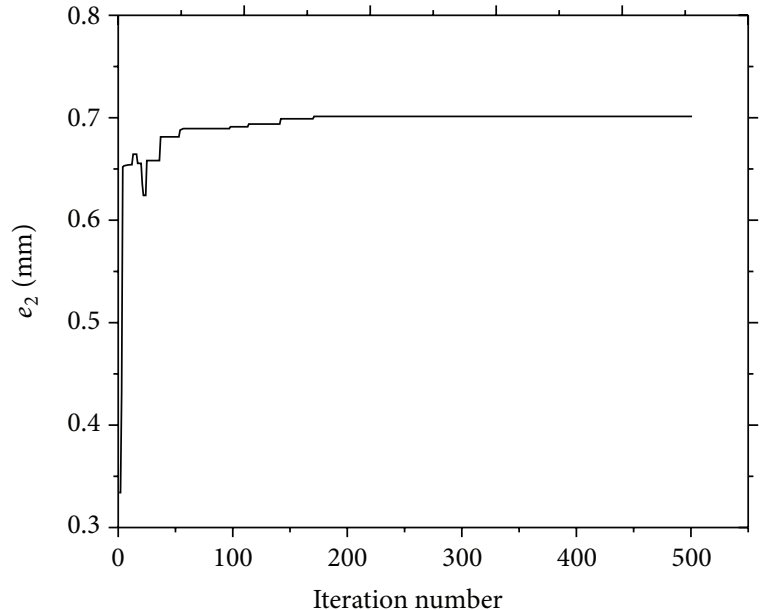

(b) $e_{2}(\mathrm{~mm})$

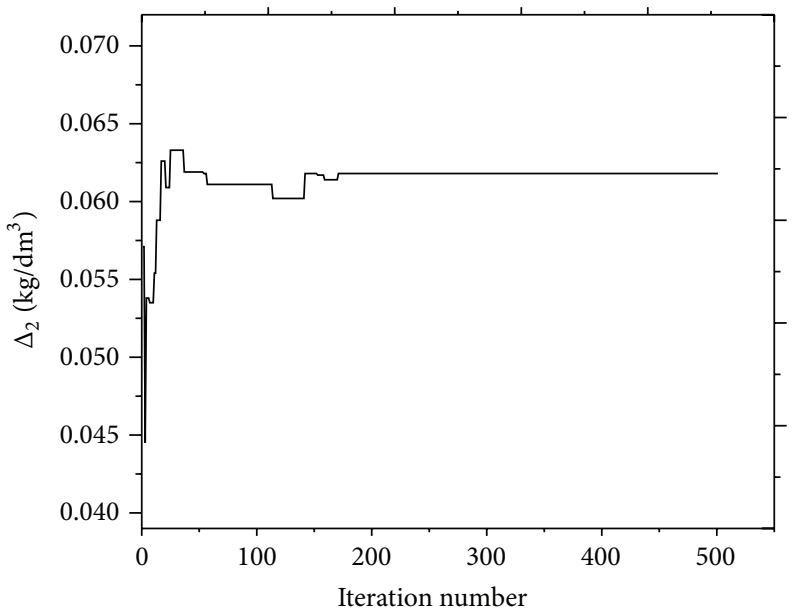

(d) $\Delta_{2}\left(\mathrm{~kg} / \mathrm{dm}^{3}\right)$

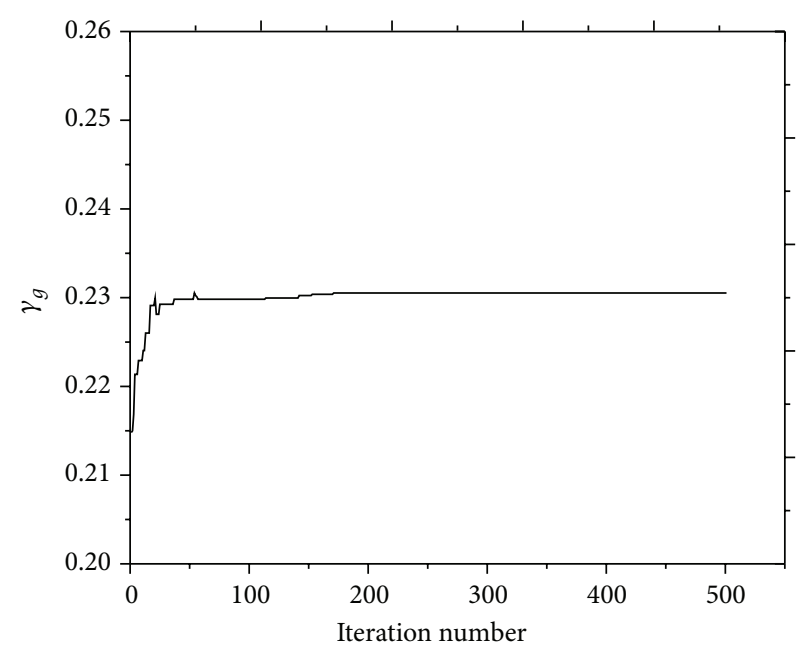

(f) $\gamma_{g}$

FIGURE 2: Convergence process of different interior ballistic parameters for single-objective problem by PSO technique. 
TABLE 4: Results of the different optimization techniques compared with the experimental date.

\begin{tabular}{lcccccc}
\hline & $v_{m}(\mathrm{~m} / \mathrm{s})$ & $P_{\max }(\mathrm{MPa})$ & $e_{1}(\mathrm{~mm})$ & $\Delta_{1}\left(\mathrm{Kg} / \mathrm{dm}^{3}\right)$ & $e_{2}(\mathrm{~mm})$ & $\Delta_{1}\left(\mathrm{Kg} / \mathrm{dm}^{3}\right)$ \\
\hline Experimental & 983.27 & 344.3 & 0.595 & 0.695 & 0.705 & 0.057 \\
GA & 985.80 & 347.2 & 0.6086 & 0.6105 & 0.6933 & 0.0608 \\
PSO & 992.52 & 345.9 & 0.6102 & 0.7078 & 0.7013 & 0.0618 \\
\hline
\end{tabular}

TABLE 5: Values of the objective functions according to the design points.

\begin{tabular}{lccc}
\hline Design points & A & B & C \\
\hline$V_{m}(\mathrm{~m} / \mathrm{s})$ & 988.12 & 970.26 & 980.21 \\
$P_{g}(\mathrm{MPa})$ & 86.132 & 82.192 & 83.372 \\
$\gamma_{g}$ & 0.2508 & 0.2536 & 0.2634 \\
\hline
\end{tabular}

to satisfy the different requirements. This section deals with multiobjective optimization model for interior ballistic propelling charge design. Three objective functions, the muzzle velocity $V_{m}$, the muzzle pressure $P_{g}$, and the energy efficiency of propelling charge $\gamma_{g}$ are considered to improve the interior ballistic performance and to satisfy the different technical requirements.

The design variables and the constraints are the same as in the single-objective problem, but the objective function is not the same. The objective function contains the following objectives.

Muzzle Velocity $\left(V_{m}\right)$. In gun design, the muzzle velocity is required to be maximized as much as possible to satisfy the tactical requirements. Hence, muzzle velocity will be the key objective function in interior ballistic design.

Muzzle Pressure $\left(P_{g}\right)$. The muzzle pressure affects the muzzle blast, the firing precision, and the initial dispersion. Hence, the muzzle pressure should be minimized as possible.

Energy Efficiency of the Charge $\left(\gamma_{g}\right)$. The energy efficiency of the charge is considered as one of the main goals in interior ballistic charge design. It should be maximized as possible.

The multiobjective model for the specific gun can be described as follows:

$$
\begin{array}{ll}
\min f(x) & =\left(-\omega_{1} V_{m},-\omega_{2} \gamma_{g}, \omega_{3} P_{g}\right) \\
\text { subject to } & V_{m} \geq 800 \mathrm{~ms}^{-1} \\
& P_{\max } \leq 350 \mathrm{MPa} \\
& 0.4 \leq \Delta \leq 0.8 \mathrm{Kg} \cdot \mathrm{dm}^{-3} \\
& 0.5 \leq \eta_{k} \leq 0.8 \\
& 0.16 \leq \gamma_{g} \leq 0.6 \\
& 0.25 \leq e_{1} \leq 0.9 \mathrm{~mm} \\
& 0.25 \leq e_{2} \leq 0.9 \mathrm{~mm},
\end{array}
$$

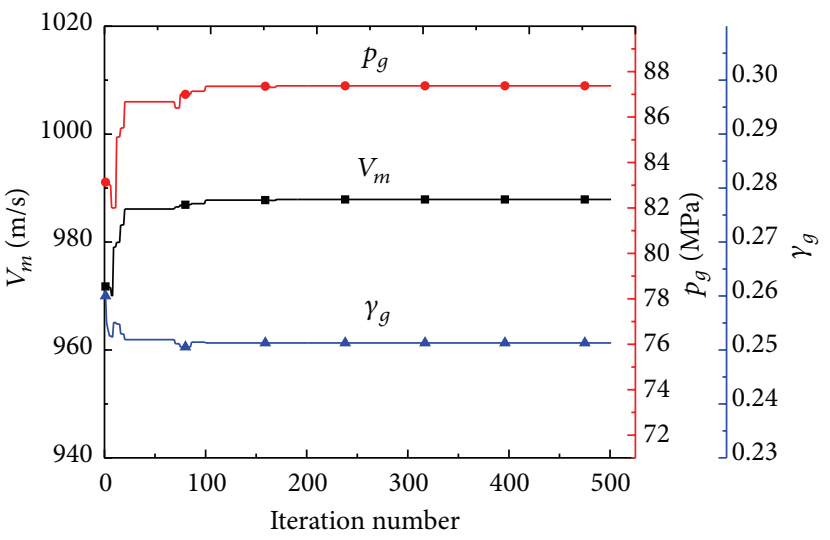

FIGURE 3: Convergence graph of the three objective functions.

where $\omega_{1}, \omega_{2}$, and $\omega_{3}$ are the weight coefficients of the objective functions determined by the gun designer according to the design requirements. For example, if the designer considers the muzzle velocity to be the main target, the weight coefficient of the muzzle velocity $\omega_{1}$ should be increased at the expense of the other coefficients $\omega_{2}$ and $\omega_{3}$.

Figure 3 shows the convergence graph for the three objective functions, muzzle velocity, muzzle pressure, and energy efficiency of the charge. It shows a good and rapid convergence to certain values determined by PSO technique. Figure 4 represents the feasible region of web thickness $e_{1}$ and propelling charge density $\Delta_{1}$ with the corresponding objective functions. Three design points marked in Figures 4(a), 4(b), and 4(c) are selected to stand for the maximum muzzle velocity, minimum muzzle pressure, and maximum energy efficiency, respectively. The values of the objective functions according to the three design points are listed in Table 5.

Based on the optimization results at point A, Figures 5 and 6 show the pressure-time and velocity-time curves for different schemes, original scheme and optimized scheme. The original scheme refers to the lumped-parameter model described in (2) without optimization. In Figure 5, the optimized scheme decreases the gradient of the pressure-time curve and the muzzle pressure. From Figure 6, the optimized scheme increases the muzzle velocity better than the original scheme. Therefore, the optimized scheme improves the interior ballistic performance and provides a better firing safety.

The feasible region shown in Figure 4 provides a large number of appropriate solutions. Obviously, the optimum solution cannot be found due to the contradictory tradeoffs among the selection conditions. Hence, an appropriate method for adapting the weight coefficients is required 


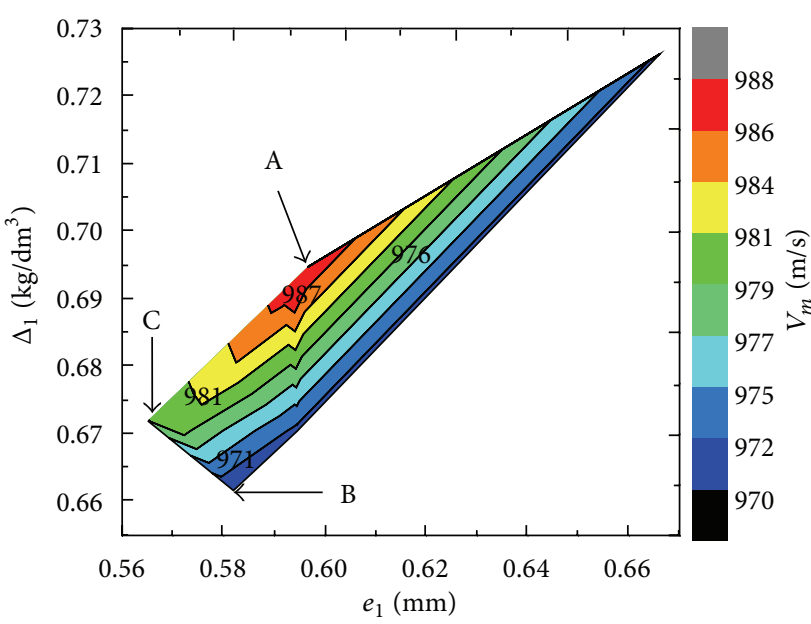

(a) Muzzle velocity

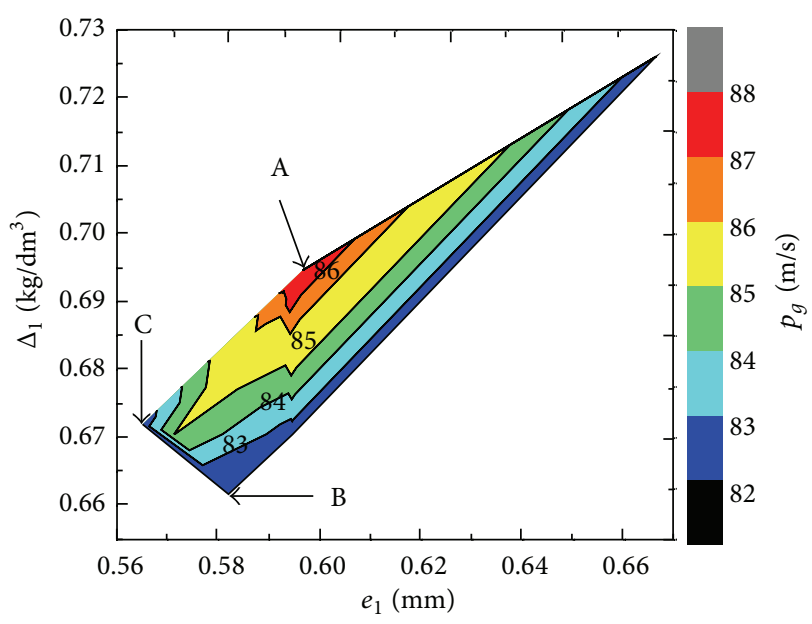

(b) Muzzle pressure

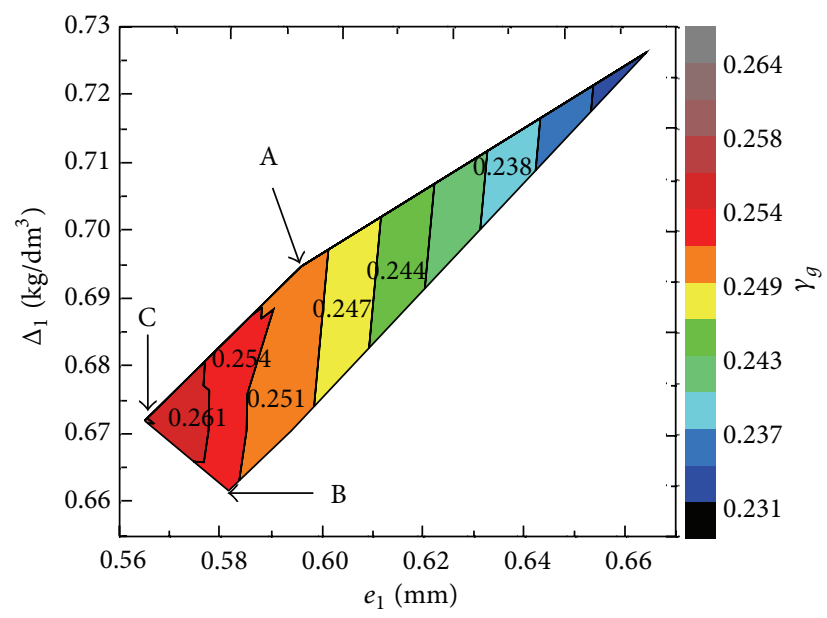

(c) Energy efficiency

FIGURE 4: Feasible region of design variables corresponding to multiobjective function.

for helping the designers of propelling charge to find the optimum solution [11].

4.2.1. Linear Analysis Method. According to the feasible region obtained by the multiobjective function, it is crucial to investigate the effect of changing the weight coefficients on the optimal solution. Hence, the linear analysis method is utilized to obtain a suitable combination of the weight coefficients. Three cases are tabulated in Table 6. One coefficient is changed linearly in each case, and the other coefficients are selected randomly in such a way that $\omega_{1}+\omega_{2}+\omega_{3}=1$, where one has the following.

Case 1. It indicates that $\omega_{1}$ is changed linearly, and $\omega_{1}$ represents the weight coefficient of muzzle velocity.

Case 2. It indicates that $\omega_{2}$ is changed linearly, and $\omega_{2}$ represents the weight coefficient of energy efficiency of the charge.

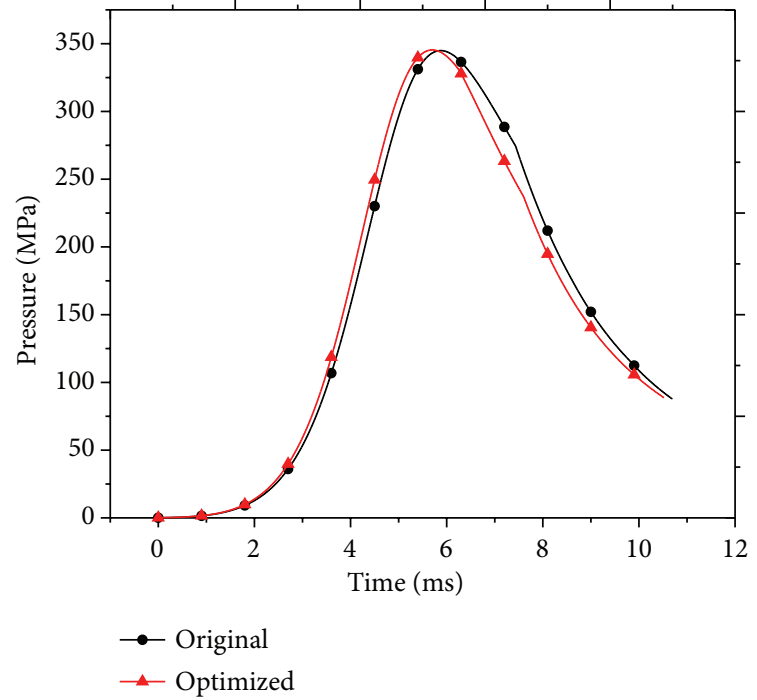

FIGURE 5: Pressure-time curve for different schemes. 
TABLE 6: Linear analysis method coefficients for the three cases.

\begin{tabular}{lccccccccc}
\hline \multirow{2}{*}{ Runs } & \multicolumn{3}{c}{ Case 1 } & \multicolumn{3}{c}{ Case 2 } & \multicolumn{3}{c}{ Case 3 } \\
& $\omega_{1}$ & $\omega_{2}$ & $\omega_{3}$ & $\omega_{1}$ & $\omega_{2}$ & $\omega_{3}$ & $\omega_{1}$ & $\omega_{2}$ \\
\hline 1 & 0 & 0.325 & 0.675 & 0.713 & 0 & 0.287 & 0.823 & 0.177 \\
2 & 0.2 & 0.648 & 0.152 & 0.535 & 0.2 & 0.265 & 0.641 & 0.159 \\
3 & 0.4 & 0.246 & 0.354 & 0.422 & 0.4 & 0.178 & 0.355 & 0.245 & 0.2 \\
4 & 0.6 & 0.023 & 0.377 & 0.213 & 0.6 & 0.187 & 0.241 & 0.159 & 0.6 \\
5 & 0.8 & 0.132 & 0.068 & 0.101 & 0.8 & 0.099 & 0.102 & 0.098 & 0.8 \\
6 & 1 & 0 & 0 & 0 & 1 & 0 & 0 & 0 \\
\hline
\end{tabular}

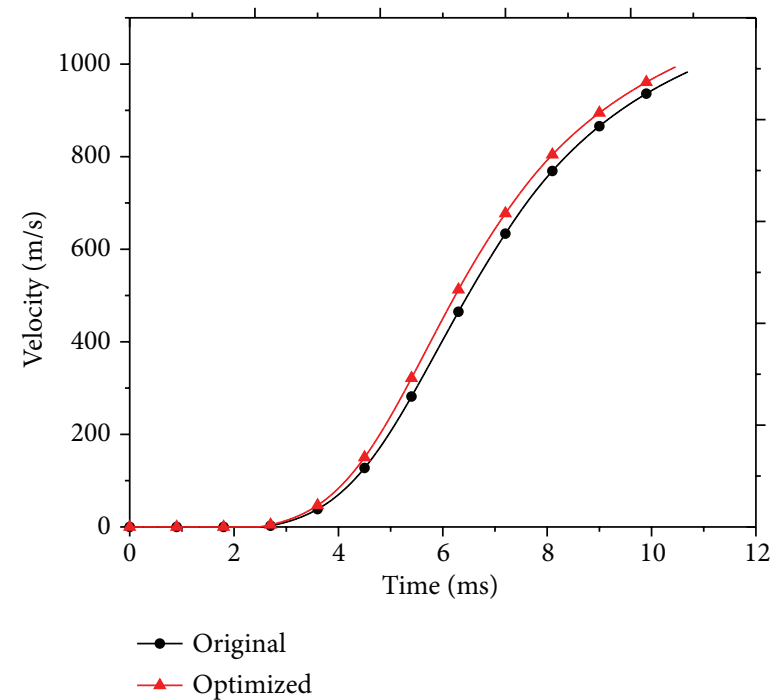

FIGURE 6: Velocity-time curve for different schemes.

Case 3. It indicates that $\omega_{3}$ is changed linearly, and $\omega_{3}$ represents the weight coefficient of muzzle pressure.

According to the multiobjective PSO method, the three objective values, $V_{m}, P_{g}$, and $\gamma_{g}$ are illustrated in Figure 7 to Figure 9 with the different weight coefficients in each case.

Through the observations of Figures 7, 8, and 9, it is obvious to conclude that the weight coefficients have a significant effect on the objective values. The highest muzzle velocity is attained at the highest value of $\omega_{1}$. The change of the energy efficiency has enormous effect on the other objective values. Accordingly, the energy efficiency can be considered as crucial index of interior ballistic performance. It can be seen from the data in Figures 7, 8, and 9 that $\omega_{1}$ is strongly recommended to take a value greater than 0.6 , $\omega_{2}$ to take a value greater than 0.1 , and $\omega_{3}$ to take a value greater than 0.1 . The designers should weigh gains and losses according to the change of the weight coefficients on the objectives to obtain the optimum performance and achieve the tactical requirements and firing safety.

\section{Conclusion}

This paper has given an account of and the reasons for the widespread use of the particle swarm optimization. PSO

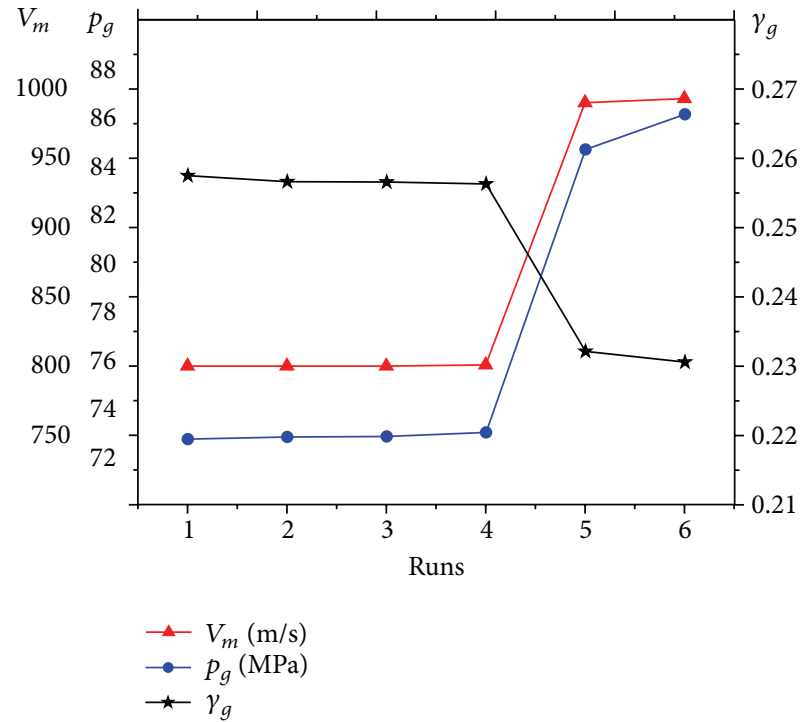

FIGURE 7: Optimal solution of different six weights of Case 1.

algorithm was developed in conjunction with interior ballistic lumped-parameter model utilizing mixed propellant. The mixed propellant is composed of granular seven-perforated propellant and tubular one-hole propellant. PSO was applied to optimize the interior ballistic propelling charge design in order to improve the interior ballistic performance. The following conclusions can be drawn from the present study.

(1) Based on the characteristics of interior ballistic propelling charge design, particle swarm optimization technique was developed with two different approaches: single-objective problem and multiobjective problem.

(2) GA and PSO techniques were applied to the single objective problem of interior ballistic propelling charge design. The optimization results were compared with the experimental data. The optimized solution showed that PSO technique has a better performance than GA. The better performance was represented in the speed of convergence and the quality of the solution.

(3) PSO with multiobjective problem provided a good opportunity for the charge's designers. The best parameters can be selected through the feasible region 


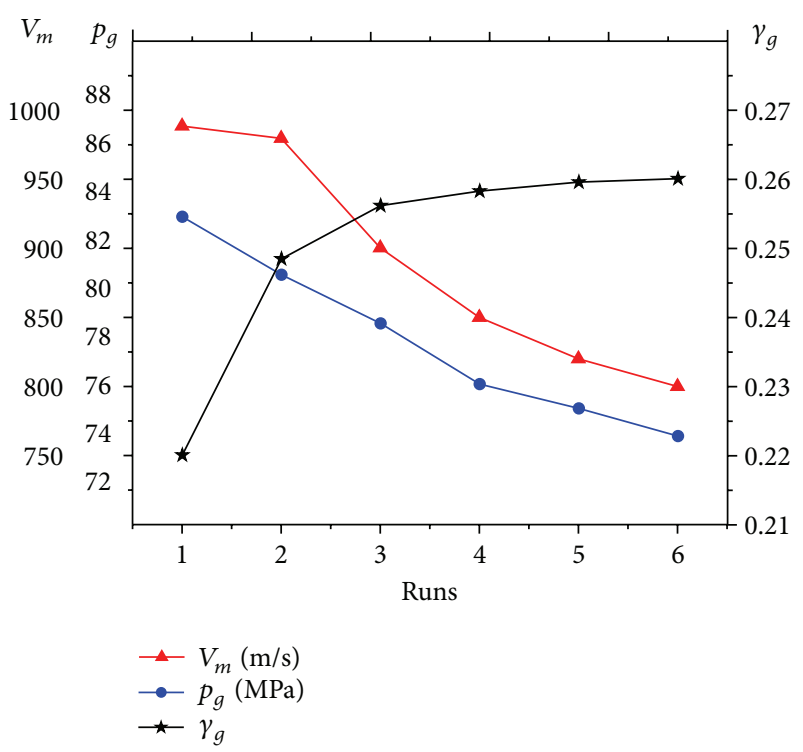

FIGURE 8: Optimal solution of different six weights of Case 2.

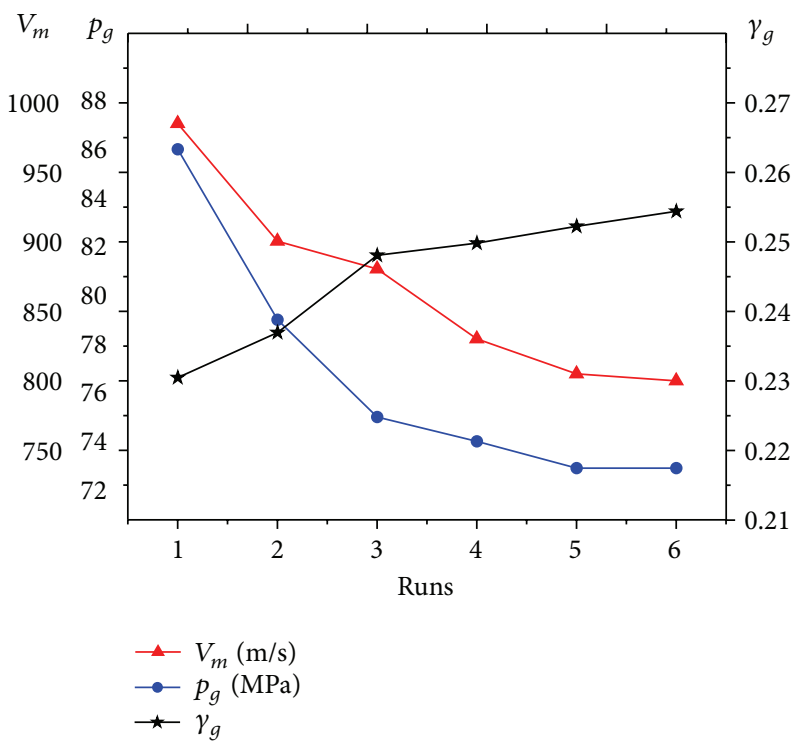

Figure 9: Optimal solution of different six weights of Case 3.

to attain the optimum solution according to the tactical requirements. Utilizing the multiobjective method improved the interior ballistic performance and assured the launch safety of the guided projectile.

(4) The linear analysis method was developed to present the most appropriate set of the weight coefficients of objectives.

\section{Conflict of Interests}

The authors declare that there is no conflict of interests regarding the publication of this paper.

\section{Acknowledgments}

The research was supported by the Research Fund for the Natural Science Foundation of Jiangsu province (BK20131348) and the Key Laboratory Fund (Grant no. 9140C300103140C30001), China.

\section{References}

[1] J. R. Gonzalez, Interior ballistics optimization [Ph.D. thesis], Kansas State University, 1990.

[2] H. S. Zhang, The optimization studies of a new ignition system and charge structure in high-velocity guns [Ph.D. thesis], Nanjing University of Science and Technology, 1998.

[3] K. Li and X. Zhang, "Multi-objective optimization of interior ballistic performance using NSGA-II," Propellants, Explosives, Pyrotechnics, vol. 36, no. 3, pp. 282-290, 2011.

[4] J. Kennedy and R. Eberhart, "Particle swarm optimization," in Proceedings of the IEEE International Conference on Neural Networks, pp. 1942-1948, December 1995.

[5] V. Kalivarapu, J.-L. Foo, and E. Winer, "Improving solution characteristics of particle swarm optimization using digital pheromones," Structural and Multidisciplinary Optimization, vol. 37, no. 4, pp. 415-427, 2009.

[6] M. Marinaki, Y. Marinakis, and G. E. Stavroulakis, "Fuzzy control optimized by a Multi-Objective Particle Swarm Optimization algorithm for vibration suppression of smart structures," Structural and Multidisciplinary Optimization, vol. 43, no. 1, pp. 29-42, 2011.

[7] Y.X. Yuan and X. B. Zhang, Multiphase Hydrokinetic Foundation of High Temperature and High Pressure, Publishing Company of Harbin Institute of Technology, Harbin, China, 2005.

[8] Y. del Valle, G. K. Venayagamoorthy, S. Mohagheghi, J.-C. Hernandez, and R. G. Harley, "Particle swarm optimization: basic concepts, variants and applications in power systems," IEEE Transactions on Evolutionary Computation, vol. 12, no. 2, pp. 171-195, 2008.

[9] W. Y. Sun and Y. Yuan, Optimization Theory and Methods Nonlinear Programming, Springer Optimization and Its Applications, Springer, 2006.

[10] A. Ravindran, K. M. Ragsdell, and G. V. Reklaitis, Engineering Optimization: Methods and Applications, John Wiley \& Sons, Hoboken, NJ, USA, 2nd edition, 2006.

[11] K. Li and X. Zhang, "Using NSGA-II and TOPSIS methods for interior ballistic optimization based on one-dimensional twophase flow model," Propellants, Explosives, Pyrotechnics, vol. 37, no. 4, pp. 468-475, 2012. 


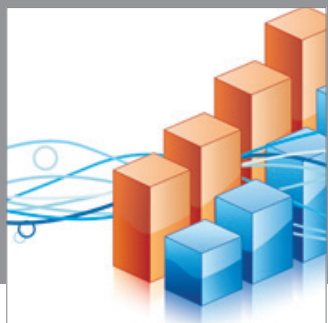

Advances in

Operations Research

mansans

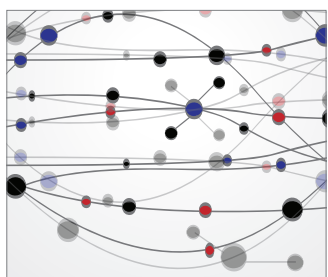

The Scientific World Journal
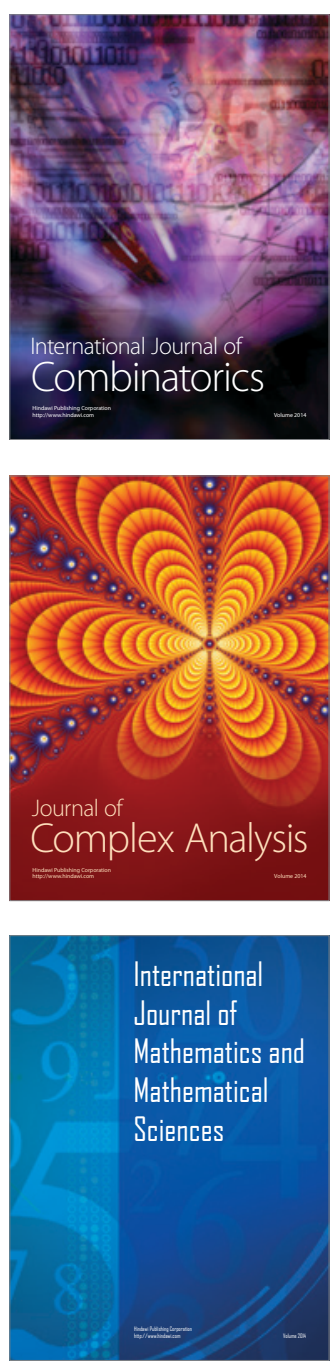
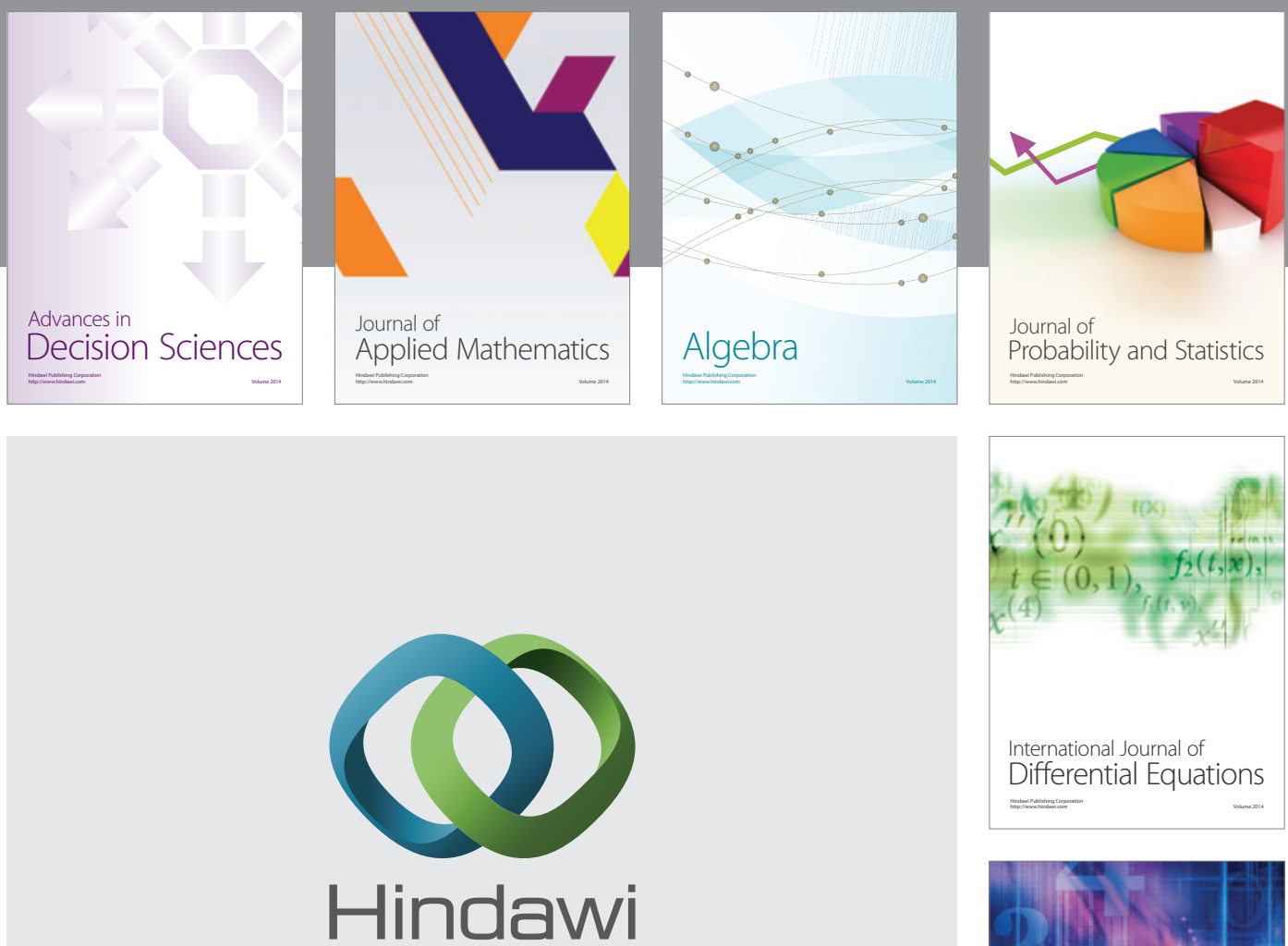

Submit your manuscripts at http://www.hindawi.com
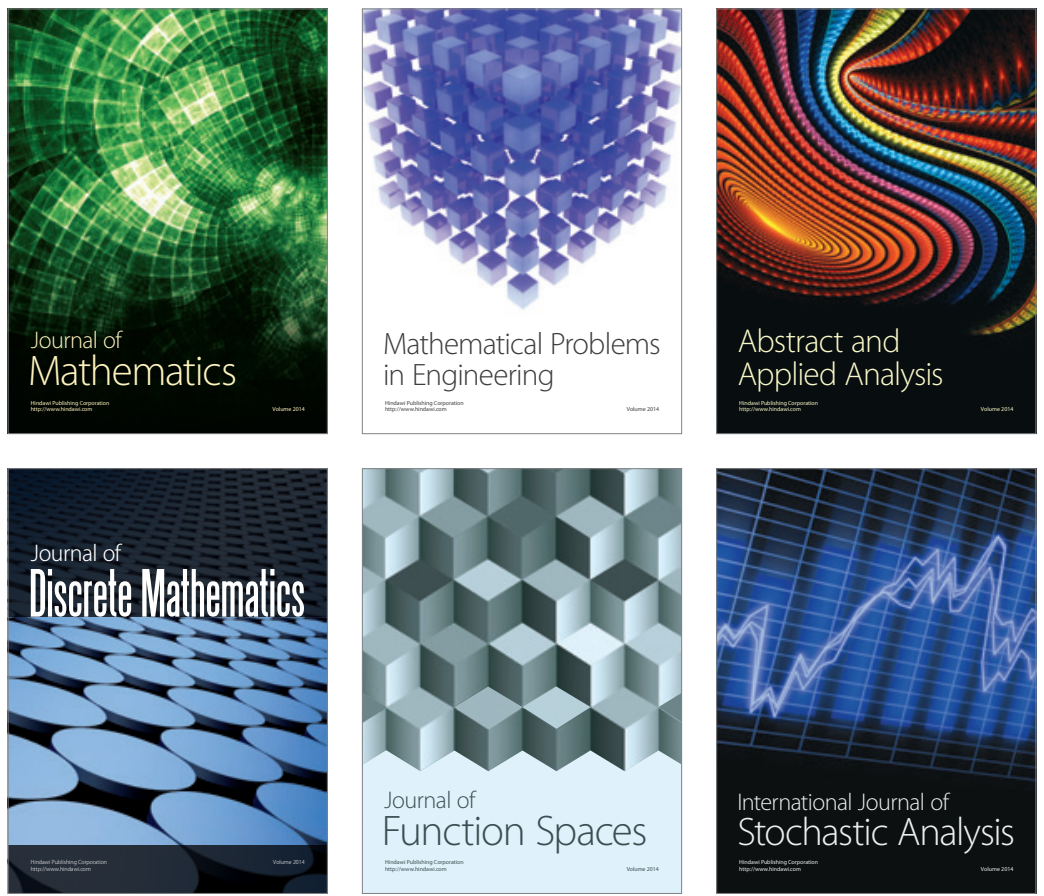

Journal of

Function Spaces

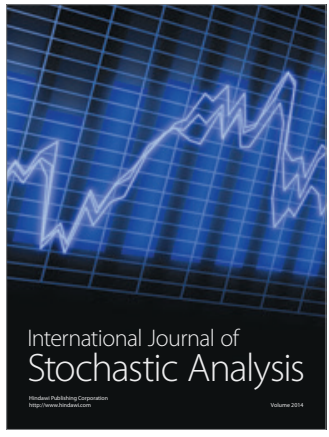

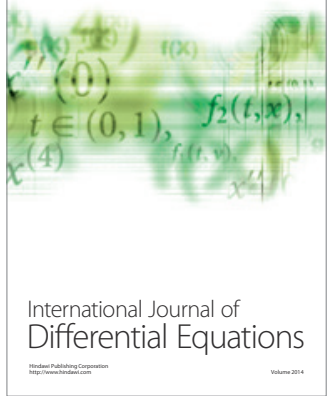
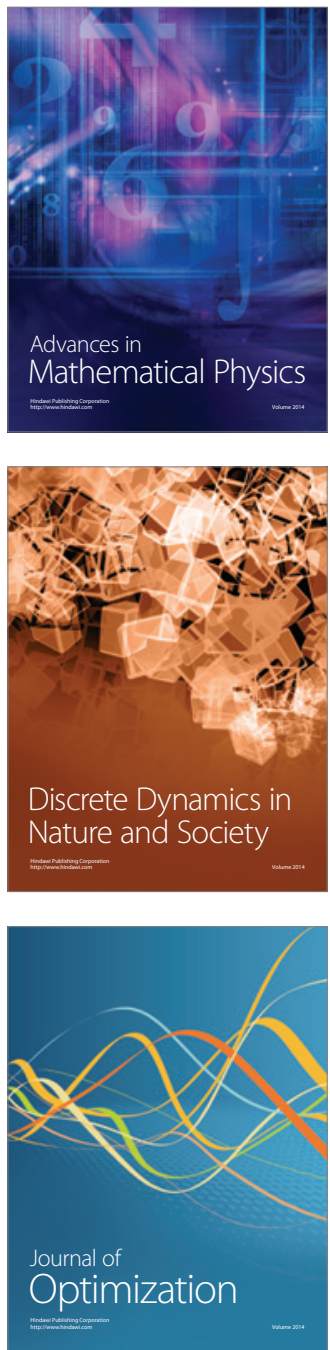